\title{
Nicotinamide Phosphoribosyl Transferase Is Increased in Osteosarcomas and Chondrosarcomas Compared to Benign Bone and Cartilage
}

\author{
ANDREW T. MERAM ${ }^{1}$, YASIR ALZUBAIDI ${ }^{2}$, JAMES COTELINGAM $^{2}$, GHALI GHALI $^{1}$, \\ LIURKA LOPEZ ${ }^{2}$, DOMENICO COPPOLA ${ }^{3}$ and RODNEY SHACKELFORD ${ }^{2}$ \\ ${ }^{1}$ Head and Neck Oncologic/Microvascular Reconstructive Surgery, \\ Department of Oral and Maxillofacial/Head and Neck Surgery, \\ Louisiana State University Health Sciences Center, Shreveport, LA, U.S.A.; \\ ${ }^{2}$ Department of Pathology and Translational Pathobiology, \\ Louisiana State University Health Sciences Center, Shreveport, LA, U.S.A.; \\ ${ }^{3}$ Department of Anatomic Pathology, H. Lee Moffitt Cancer Center and Research Institute, Tampa, FL, U.S.A.
}

\begin{abstract}
Background/Aim: Primary bone neoplasms include osteosarcomas (OS), chondrosarcomas (CS), and giant cell tumors (GCT). Nicotinamide phosphoribosyl transferase (NAMPT) catalyzes the rate-limiting step of nicotinamide adenine dinucleotide synthesis and is increased in multiple tumor types. In malignancies, NAMPT expression often correlates positively with tumor grade, chemotherapy resistance, and metastatic potential. Materials and Methods: Tissue microarray was used to examine NAMPT expression in benign bone and cartilage, GCTs, OS, and different CS grades. Results: For the first time, we showed that NAMPT expression was increased in GCTs and OS compared to benign bone, and in CS compared to benign cartilage. Its expression also increased with higher CS grade. Conclusion: Our data indicate that NAMPT plays a role in bone sarcomas and GCTs, and its higher expression may contribute to increased tumor aggressiveness.
\end{abstract}

Malignancies of the bones and joints are rare, comprising $0.2 \%$ of all human neoplasms. In the United States, approximately 3,450 were diagnosed in 2017 , while in the same year these malignancies caused 1,590 deaths $(1,2)$. Among these malignancies, osteosarcomas (OS) and chondrosarcomas (CS) constitute $36 \%$ and $20-25 \%$ of bone and joint malignancies, respectively (1-3). While OS can

This article is freely accessible online.

Correspondence to: Rodney Edwin Shackelford, Department of Pathology and Translational Pathobiology Louisiana State University Health Sciences Center, Shreveport, LA, U.S.A. E-mail: rshack@1suhsc.edu

Key Words: Osteosarcoma, chondrosarcoma, NAMPT. occur in patients of all ages, it exhibits a bimodal distribution, with disease peaks at 15-19 years and 75-79 years (1-3). Although most cases are sporadic, factors such as radiation exposure, Paget's disease of the bone, and genetic susceptibilities increase OS risk (1-4). They commonly present in the long bones at areas of rapid bone growth, typically adjacent to the metaphyseal growth plate, commonly at the proximal humerus and tibia, and distal femur (1-4). OS is more common in males and confers different survival rates by different ages and anatomic locations (1-4). In adolescents and children, the 5-year survival rate is approximately 55$75 \%$, with older individuals having an approximately 57\% 5year survival rate that falls with increasing age (1-4). Histologically, OS exhibit malignant pleomorphic cells with an osteogenic differentiation that produces osteoid, typically in irregular trabeculae (1-4). While malignant bone is always present, the matrix of conventional OS may have chondroid, fibroblastic, or cartilaginous features (1-4). Less common OS subtypes include telangiectatic, small-cell, periosteal, paraosteal, extraskeletal, and secondary OS (1-4).

CS are adult bone sarcomas and often present as slowgrowing heterogenous malignancies in individuals between 30 and 60 years old $(2-3,5)$. CS are associated with a 5-year survival rate of approximately $70 \%$, are slightly more common in males, and commonly occur in the lower limbs, pelvis, sternum, ribs, and clavicle $(2-3,5)$. CS is often graded on a I-III scale, with $90 \%$ graded I-II and 5-10\% grade III, which carry significant metastatic potential (2-3, $5)$. CS is classified as primary if not associated with a preexisting chondroid lesion and secondary if they it is, often with an enchondroma or osteochondroma (2-3, 5). Risk factors of CS include Ollier and Maffucci diseases, and radiation exposure (7-9). Histologically, CS typically exhibit a hyaline cartilaginous matrix with admixed malignant 
chondrocytes $(2,5)$. Grade I CS have a relatively low cellularity and cytonuclear atypia with a mainly chondroid matrix and occasional binucleated cells $(2,5)$. Grade II-III exhibit increased cellularity, cytonuclear atypia, matrix mucomyxoid degeneration, and increased binucleated cells, mitotic figures, and metastatic potential $(2,5)$. Less common CS histological types include dedifferentiated CS, mesenchymal CS, clear-cell CS, and extraskeletal myxoid CS (5). Both OS and CS typically present as slow-growing, painful masses that do not resolve over time $(2,4-6)$.

Giant cell tumors (GCT) are relatively common benign bone tumors and comprise $5 \%$ of primary bone tumors $(1,2)$. They commonly occur in the metaphyseal region of long bones, showing extensive bony destruction by a vascular tumor with numerous osteoclast-like giant cells with many nuclei, mononuclear spindle cells, and often acute hemorrhage with hemosiderin deposition and focal necrosis $(1,2)$.

At the molecular level, OS often carry p53 and retinoblastoma gene mutations $(2,6,10-13)$. Additionally, underexpression of dedicator of cytokinesis 5, cyclindependent kinase inhibitor 1, limbic system-associated membrane protein, and tumor necrosis factor receptor superfamily member $10 \mathrm{~A} / \mathrm{D}$, and overexpression of runtrelated transcription factor 2, ATP-dependent DNA helicase Q4, secreted phosphoprotein, and integrin binding sialoprotein bone sialoprotein is common (12-13). OS also often exhibit either increased telomerase expression or increased alternative lengthening of telomeres, contributing to their malignant growth (14). CS often carry isocitrate dehydrogenase mutations resulting in the production of the oncometabolite $\delta$-2-hydroxyglutarate $(2,5,13)$. Additionally, alterations in retinoblastoma tumor pathway signaling, p53 mutations, and phosphatidylinositol-4,5-bisphosphate 3-kinase/mammalian target of rapamycin kinase activation are common in CS $(2,5,15-18)$. Similarly, to OS, CS frequently overexpress telomerase and carry telomerase promoter mutations that promote metastasis (19). GCTs commonly exhibit mutations of $\mathrm{H} 3$ histone family member $3 \mathrm{~A}$ and receptor activator of nuclear factor kappa-light ligand, accompanied by telomeric association, where chromosomal ends form dicentric or multicentric ring chromosomes (20).

Nicotinamide phosphoribosyl transferase (NAMPT) catalyzes the rate-limiting step of nicotinamide adenine dinucleotide synthesis and exhibits increased expression in several malignancies, including gastric, thyroid, urothelial, renal, cervical, oral and cervical squamous cell carcinomas, rhabdomyosarcomas, and leiomyosarcomas (21-26). NAMPT expression also increases with increasing grade of cervical dysplasia (26). NAMPT expression has not been previously examined in OS or CS to our knowledge. Here we used microarray technology to examine NAMPT protein levels in osteoblastic, chondroblastic, and telangiectatic OS, grade I-III CS, and GCTs of the bone.
Table I. Relative nicotinamide phosphoribosyltransferase staining in the two tissue microarrays comparing benign bone and cartilage to osteosarcomas and different grades of chondrosarcomas and giant cell tumors of the bone.

\begin{tabular}{lrcl}
\hline Tissue & $\begin{array}{c}\text { Number of } \\
\text { type }\end{array}$ & $\begin{array}{c}\text { Average IHC } \\
\text { samples }\end{array}$ & $\begin{array}{l}\text { SEM } \\
\text { score }\end{array}$ \\
\hline Benign & & & \\
$\quad$ Bone & 25 & 0.54 & 0.25 \\
$\quad$ Cartilage & 7 & 0 & 0 \\
Osteosarcoma & & & \\
$\quad$ Telangiectatic & 8 & $5.63^{\mathrm{a}}$ & 0.61 \\
Osteoblastic & 52 & $6.73^{\mathrm{a}}$ & 1.23 \\
Chondroblastic & 4 & $5.0^{\mathrm{a}}$ & 0.58 \\
Chondrosarcoma & & & \\
$\quad$ Well-differentiated & 43 & $1.79^{\mathrm{b}}$ & 0.84 \\
Intermediately differentiated & 10 & $3.90^{\mathrm{bc}}$ & 0.84 \\
$\quad$ Poorly differentiated & 6 & $9.0 \mathrm{~b}^{\mathrm{c}}$ & 0 \\
Giant cell tumor & 36 & $7.81^{\mathrm{a}}$ & 0.36 \\
\hline
\end{tabular}

Significantly different from: ${ }^{\mathrm{a} B o n e},{ }^{\mathrm{b}}$ cartilage, ${ }^{\mathrm{c}}$ well-differentiated chondrosarcoma at $p<0.05$.

\section{Materials and Methods}

Tissue microarray (TMA). Two TMAs, OS208 and T261, were purchased from US Biomax, Inc. (Rockville, MD, USA). Both TMAs were interrogated by an antibody to NAMPT. Together the two TMAs contained 25 samples of benign bone, 7 of benign cartilage, eight of telangiectatic OS, 53 of OC, four of chromoblastic OS, 36 of GCT, and 43, 10, and six samples of well, moderately, and poorly differentiated CS, respectively. All tissue samples in the TMAs were $1.5 \mathrm{~mm}$ in diameter.

NAMPT immunohistochemistry (IHC). The concentration of the primary antibody to NAMPT was optimized to normal kidney as control tissue, the where concentrations of the primary and secondary antibodies were titrated until optimal immunohistochemical NAMPT protein identification was achieved. The staining of the TMAs was performed at the Tissue Core Histology Lab Facility at the Moffitt Cancer Center. The microarray slides were stained using a Ventana Discovery XT automated system (Ventana Medical Systems, Tucson, AZ, USA) as per the manufacturer's protocol with proprietary reagents. Briefly, the slides were deparaffinized on the automated system with EZ Prep solution (Ventana Medical Systems). The heat-induced antigen retrieval method was used in Cell Conditioning 1 (Ventana Medical Systems). Mouse monoclonal antibody to human NAMPT (Enzo Life Sciences, Plymouth Meeting, PA, USA) was used at a 1:1,000 concentration in Dako antibody diluent (Dako, Carpentaria, CA, USA) and incubated with TMAs for $60 \mathrm{~min}$. Ventana anti-mouse secondary antibodies were used for $16 \mathrm{~min}$. The detection system used was the Ventana OmniMap kit. Slides were then dehydrated and cover-slipped per standard laboratory protocol.

Evaluation of NAMPT staining. Relative NAMPT protein expression was determined as immunostain intensity scored on a 0 to 3 scale as follows: no staining: 0 , light staining: 1 , moderate staining: 2, and heavy staining: 3 . The percentage of cells stained 


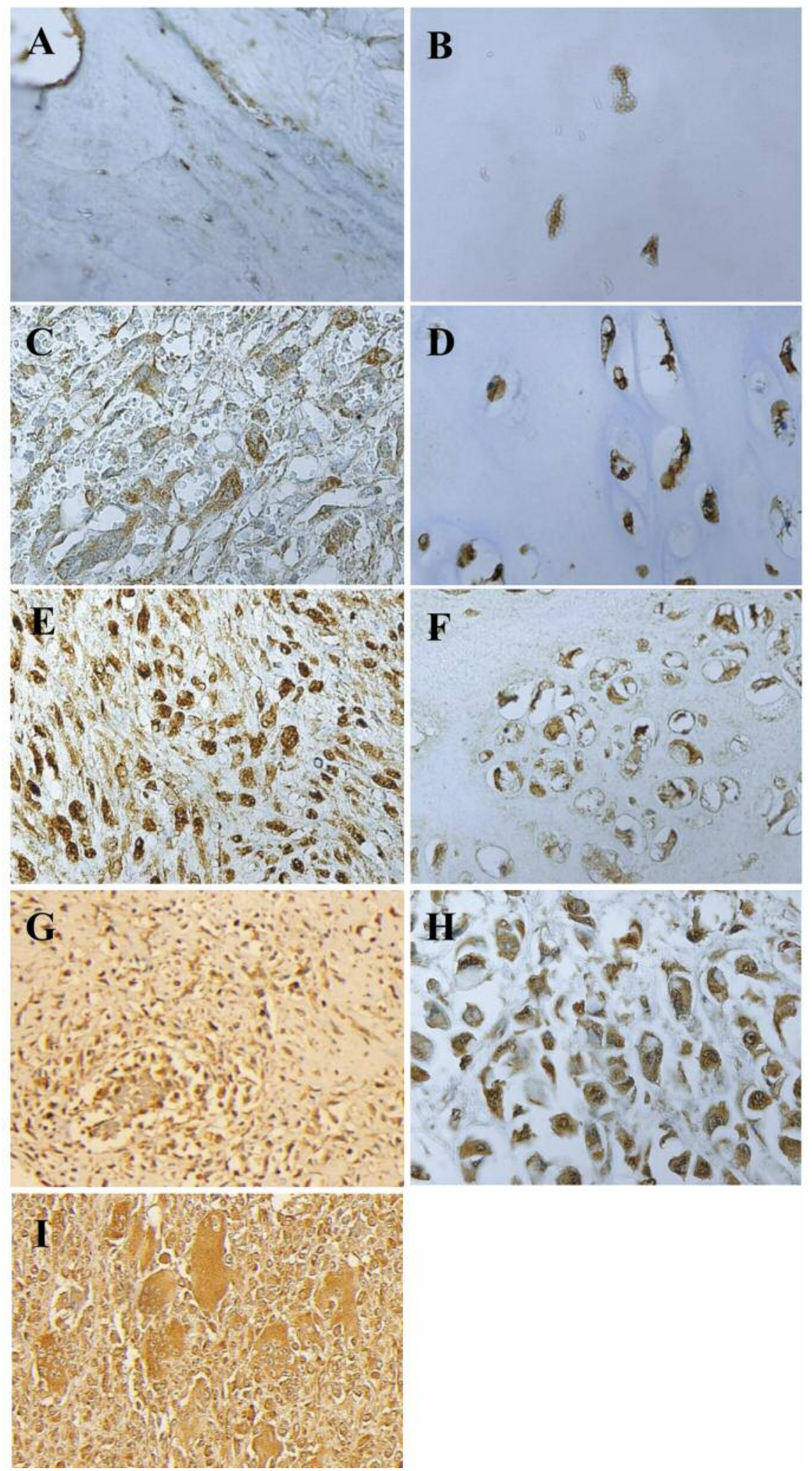

Figure 1. Representative immunostaining of nicotinamide phosphoribosyl transferase in benign bone (A), benign cartilage (B), telangiectatic osteosarcoma $(C)$, grade I chondrosarcoma $(D)$, osteoblastic osteosarcoma $(E)$, grade II chondrosarcoma $(F)$, chondroblastic osteosarcoma $(G)$, grade III chondrosarcoma $(H)$ and giant cell tumor of the bone (I). Original magnification $\times 400$. 
was measured, with no detectable staining as $0,1-33 \%$ as $1,34-66 \%$ as 2 , and $67-100 \%$ as 3 . The final IHC score was the product of the percentage of cells stained multiplied by the intensity score, allowing for a minimal score of 0 and a maximal score of 9 . Nuclear and cytoplasmic NAMPT staining was seen in all tissue samples examined. Therefore, NAMPT staining in both the nuclear and cytoplasmic compartments were measured and quantified as one.

Statistical analysis of TMA results. The standard error of the mean (SEM) IHC score was calculated using the standard deviation for the staining scores of each tumor type and dividing this number by the square root of the sample size $(22,26)$. The significance of differences in all the experiments was calculated using prism software version 5.02 (Graph Pad Inc., San Diego, CA, USA).

\section{Results}

Following IHC processing none of the tissue samples on the TMAs were lost. The number of cases examined, the IHC scoring result, and the SEM of each data set are given in Table I. Examples of benign tissues and different OS types, CS grades, and giant cell tumor of the bone are shown in Figure 1. NAMPT was significantly increased in each OS type compared to benign bone tissue and was also significantly increased in CS compared to benign cartilage, with significant differences being found between benign bone and all three OS types and between benign cartilage and all three CS grades. Significant differences were also found between welldifferentiated and intermediate and poorly differentiated CS. Lastly, differences in NAMPT expression between benign bone and GCTs of the bone were also significant (Figure 1).

\section{Discussion}

Here we have shown for the first time that NAMPT is increased in OS, CS, and GCTs. In the CS, NAMPT expression increased with CS grade and was greatest in grade III (poorly differentiated) CS. High-grade CS has an increased metastatic potential and confer a worse prognosis $(27,28)$. Similarly, higher NAMPT expression is seen with increasing grade of cervical dysplasia, leiomyosarcoma, oral and cervical squamous cell carcinoma, and Fuhrman grade in renal cell carcinoma $(22-24,26)$. Additionally, increased NAMPT expression was shown to confer a worse prognosis in endometrial adenocarcinoma and to confer resistance to chemotherapeutic agents $(21,29,30)$. Lastly, higher NAMPT expression positively correlated with more aggressive rhabdomyosarcoma subtypes, compared to less aggressive subtypes (26). Our data that NAMPT expression increases with higher grade of CS add support to the hypothesis that NAMPT overexpression in a malignancy confers a more aggressive clinical course and a worse prognosis.

Interestingly, NAMPT expression was high in GCT of the bone (Table I, Figure 1). The great majority of these tumors are benign and only exert locally destructive effects (2, 4-6,
20). High NAMPT expression was seen in renal oncocytoma, which is also usually a benign lesion (23). Based on this, it appears likely that increased NAMPT expression is a reflection of an increased cellular demand for nicotinamide adenine dinucleotide and is not a specific marker for malignant transformation. Further work will be needed to clarify this hypothesis.

Here we have shown for the first time that NAMPT is increased in GCT of the bone and in two bone sarcoma types. We also show for the first time that NAMPT expression increases with higher CS grades. Taken together our data help confirm that high NAMPT expression is seen in a wide variety of malignancies, including sarcomas.

\section{Conflicts of Interest}

The Authors have no conflict of interest to report in regard to this study.

\section{Authors' Contributions}

All Authors assisted in the writing of the manuscript. Liurka Lopez was also consulted on the histology and pathology of OS and CS. James Cotelingam also took the photographs and Domenico Coppola oversaw the process of making the IHC staining. Lastly, Rodney Shackelford assembled the final manuscript and performed the statistical amylases.

\section{Acknowledgements}

The Authors thank the Histology Section of the Tissue Core at the Moffitt Cancer Center and Research Institute for support in performing the IHC stains. We also thank Nicole Maxwell for her assistance in editing this manuscript.

\section{References}

1 American Cancer Society: Cancer Facts \& Figures. 2017.

2 Fletcher CM, Unni KK and Mertens F (eds.): World Health Organization Classification of Tumours. Pathology and Genetics of Tumours of Soft Tissue and Bone. IARC Press: Lyon 2002.

3 Whelan J, McTiernan A, Cooper N, Wong YK, Francis M, Vernon $\mathrm{S}$ and Strauss SJ: Incidence and survival of malignant bone sarcomas in England. Int J Cancer 131: E508-E517, 2012. PMID: 21913189. DOI: 10.1002/ijc.26426

4 Savage S and Mirabello L: Using epidemiology and genomics to understand osteosarcoma etiology. Sarcoma 2011: 548151, 2011. PMID: 21437228. DOI: $10.1155 / 2011 / 548151$

5 Chow WA: Chondrosarcoma: Biology, genetics and epigenetics. F1000Res 7: F1000-1826, 2018. PMID: 30519452. DOI: 10.12688/f1000research.15953.1

6 Gonzalez AL and Cates JM: Osteosarcoma: Differential diagnostic considerations. Surg Pathol Clin 5: 117-146, 2012. PMID: 26837918. DOI: 10.1016/j.path.2011.07.011

7 Ollier M: Dyschondroplasie. Lyon Med 93: 23-25, 1900.

8 Maffucci A: Di un caso encondroma ed angioma multiplo. Mov Med Chir 3: 399-412 and 565-575, 1881. 
9 Thiagarajan A and Iyer NG: Radiation-induced sarcomas of the head and neck. World J Clin Oncol 5: 973-981, 2014. PMID: 25493233. DOI: 10.5306/wjco.v5.i5.973

10 Walkley CR, Qudsi R, Sankaran VG, Perry JA, Gostissa M, Roth SI, Rodda SJ, Snay E, Dunning P, Fahey FH, Alt FW, McMahon AP and Orkin SH: Conditional mouse osteosarcoma, dependent on $\mathrm{p} 53$ loss and potentiated by loss of $\mathrm{Rb}$, mimics the human disease. Genes Dev 22: 1662-1676, 2008. PMID: 18559481. DOI: $10.1101 / \mathrm{gad} .1656808$

11 Donehower LA, Harvey M, Slagle BL, McArthur MJ, Montgomery CA Jr., Butel JS and Bradley A: Mice deficient for p53 are developmentally normal but susceptible to spontaneous tumours. Nature 356: 215-221, 1992. PMID: 1552940. DOI: $10.1038 / 356215 \mathrm{a} 0$

12 Sadikovic B, Yoshimoto M, Chilton-MacNeill S, Thorner P, Squire JA and Zielenska M: Identification of interactive networks of gene expression associated with osteosarcoma oncogenesis by integrated molecular profiling. Hum Mol Genet 18: 1962-1975, 2009. PMID: 19286668. DOI: $10.1093 / \mathrm{hmg} / \mathrm{ddp} 117$

13 Sadikovic B, Thorner P, Chilton-Macneill S, Martin JW, Cervigne NK, Squire J and Zielenska M: Expression analysis of genes associated with human osteosarcoma tumors shows correlation of RUNX2 overexpression with poor response to chemotherapy. BMC Cancer 10: 202, 2010. PMID: 20465837. DOI: $10.1186 / 1471-2407-10-202$

14 Ulaner GA, Hoffman AR, Otero J, Huang HY, Zhao Z, Mazumdar M, Gorlick R, Meyers P, Healey JH and Ladanyi M: Divergent patterns of telomere maintenance mechanisms among human sarcomas: sharply contrasting prevalence of the alternative lengthening of telomeres mechanism in Ewing's sarcomas and osteosarcomas. Genes Chromosomes and Cancer 41: 155-162, 2004. PMID: 15287028. DOI: 10.1002/gcc.20074

15 de Andrea CE, Reijnders CM, Kroon HM, de Jong D, Hogendoorn PC, Szuhai K and Bovée JV: Secondary peripheral chondrosarcoma evolving from osteochondroma as a result of outgrowth of cells with functional EXT. Oncogene 31: 1095104, 2012. PMID: 21804604. DOI: 10.1038/onc.2011.311

16 Schrage YM, Lam S, Jochemsen AG, Cleton-Jansen AM, Taminiau AH, Hogendoorn PC and Bovée JV: Central chondrosarcoma progression is associated with $\mathrm{pRb}$ pathway alterations: CDK4 down-regulation and p16 overexpression inhibit cell growth in vitro. J Cell Mol Med 13: 2843-2852, 2009. PMID: 18624751. DOI: 10.1111/j.1582-4934.2008. 00406.x

17 Blasenbreu S, Baretton GB, Bender C, Haas CJ, Diebold J and Löhrs U: TP53 gene aberrations in chondromatous neoplasms: Correlation with immunohistochemical p53 accumulation and MDM2 expression. Verh Dtsch Ges PathoI 82: 284-289, 1998. PMID: 10095448

18 Zhang YX, van Oosterwijk JG, Sicinska E, Moss S, Remillard $\mathrm{SP}$, van Wezel T, Bühnemann C, Hassan AB, Demetri GD, Bovée JV and Wagner AJ: Functional profiling of receptor tyrosine kinases and downstream signaling in human chondrosarcomas identifies pathways for rational targeted therapy. Clin Cancer Res 19: 3796-807, 2013. PMID: 23714727. DOI: $10.1158 / 1078-0432 . C C R-12-3647$

19 Lin Y, Seger N, Chen Y, Hesla AC, Wejde J, Ghaderi M, Tsagkozis $\mathrm{P}$, Larsson $\mathrm{O}$ and Haglund F: hTERT promoter mutations in chondrosarcomas associate with progression and disease-related mortality. Mod Pathol 31: 1834-1841, 2018. PMID: 30065261. DOI: 10.1038/s41379-018-0098-3
20 Noh BJ and Park YK: Giant cell tumor of bone: Updated molecular pathogenesis and tumor biology. Hum Pathol 81: 1-8, 2018. PMID: 29944971. DOI: 10.1016/j.humpath.2018.06.017

21 Shackelford RE, Mayhall K, Maxwell NM, Kandil E and Coppola D: Nicotinamide phosphoribosyltransferase in malignancy: A review. Genes Cancer 4: 447-456, 2013. PMID: 24386506. DOI: $10.1177 / 1947601913507576$

22 Vora M, Ansari J, Shanti RM, Veillon D, Cotelingam J, Coppola $\mathrm{D}$ and Shackelford RE: Increased nicotinamide phosphoribosyltransferase in rhabdomyosarcomas and leiomyosarcomas compared to skeletal and smooth muscle tissue. Anticancer Res 36: 503-507, 2016. PMID: 26851003.

23 Shackelford RE, Abdulsattar J, Wei EX, Cotelingam J, Coppola $\mathrm{D}$ and Herrera GA: Increased nicotinamide phosphoribosyltransferase and cystathionine- $\beta$-synthase in renal oncocytomas, renal urothelial carcinoma and renal clear cell carcinoma. Anticancer Res 37: 3423-3427, 2017. PMID: 28668830. DOI: 10.21873/anticanres.11709

24 Patel S, Ansari J, Meram A, Abdulsattar J, Cotelingam J, Coppola D, Ghali $G$ and Shackelford RE: Increased nicotinamide phosphoribosyltransferase and cystathionine-betasynthase in oral cavity squamous cell carcinomas. Int J Clin Exp Pathol 10: 702-707, 2017.

25 Turbat-Herrera EA, Kilpatrick MJ, Chen J, Meram AT, Cotelingam J, Ghali G, Kevil CG, Coppola D and Shackelford RE: Cystathione $\beta$-synthase is increased in thyroid malignancies. Anticancer Res 38: 6085-6090, 2018. PMID: 30396922. DOI: 10.21873/anticanres.12958

26 Vora M, Alattia LA, Ansari J, Ong M, Cotelingam J, Coppola D and Shackelford R: Nicotinamide phosphoribosyl transferase: A reliable marker of progression in cervical dysplasia. Anticancer Res 37: 4821-4825, 2017. PMID: 28870901. DOI: 10.21873/ anticanres.11889

27 van Praag Veroniek VM, Rueten-Budde AJ, Ho V and Dijkstra PDS: Incidence, outcomes and prognostic factors during 25 years of treatment of chondrosarcomas. Surg Oncol 27: 402-408, 2018. PMID: 30217294. DOI: 10.1016/j.suronc.2018.05.009

28 Andreou D, Ruppin S, Fehlberg S, Pink D, Werner M and Tunn PU: Survival and prognostic factors in chondrosarcoma: Results in 115 patients with long-term follow-up. Acta Orthop 82: 74955, 2011. PMID: 22066552. DOI: 10.3109/17453 674.2011 .636668

29 Tian W, Zhu Y, Wang Y, Teng F, Zhang H, Liu G, Ma X, Sun D, Rohan $\mathrm{T}$ and Xue F: Visfatin, a potential biomarker and prognostic factor for endometrial cancer. Gynecol Oncol 129: 505-512, 2013. PMID: 23438672. DOI: 10.1016/j.ygyno.2013. 02.022

30 Wang B, Hasan, MK, Alvarado E, Yuan H, Wu H and Chen WY: NAMPT overexpression in prostate cancer and its contribution to tumor cell survival and stress response. Oncogene 30: 907921, 2011. PMID: 20956937. DOI: 10.1038/onc.2010.468
Received January 27, 2019

Revised February 26, 2019

Accepted February 27, 2019 